\title{
Power allocation and relay selection for energy efficient cooperation in wireless sensor networks with energy harvesting
}

\author{
Yin $\mathrm{Wu}^{1 *}$, Wenbo Liư ${ }^{2}$ and Kaiyu $\mathrm{Li}^{2}$
}

\begin{abstract}
In this paper, we investigate a joint power allocation and relay selection scheme for energy efficient cooperation in energy harvesting-wireless sensor networks (EH-WSN). We mainly propose a simple heuristic algorithm to improve the energy efficiency of each node in a clustering based EH-WSN. First, the effect of cooperative communication is evaluated, and then, the energy sustainability of every node is taken into account, finally, we formulate an online optimization problem which can be solved near optimally with low computational complexity. Extensive simulation results are presented to show the outstanding performance of our proposed scheme in nodes' transmitting power allocation and average working utility. Therefore, this joint optimization algorithm has a promising future in real applications.
\end{abstract}

Keywords: Cooperative communication, Energy harvesting, Wireless sensor network, Transmitting power control, Relay selection

\section{Introduction}

Nowadays, a new class of wireless sensor network that harvest energy from the environment (solar, wind, vibration, etc.) is emerging. Because of its intrinsic capability of self-sustainability, a lot of researches have been done on it; many of them focus on the high-efficient energy utilization [1-3]. As for this energy harvesting-wireless sensor networks (EH-WSN), the main challenge is to maximize its working performance under energy harvesting constraints, so studies on the MAC protocol, routing protocol, and cross layer optimization also obtain lots of achievements [4-10].

Recently, the use of cooperative communication in EH-WSN has attracted some interest [11, 12]. For example, Kuang-Hao Liu has investigated a battery-aware relay selection scheme, which is called BARS, for energy harvesting $(\mathrm{EH})$ relays with finite energy storage [13]. It takes into account both channel state information and battery status in choosing the cooperating relay. The analysis result shows that the number of potential relays

\footnotetext{
* Correspondence: wuyin@njfu.edu.cn

${ }^{1}$ College of Information Science and Technology, Nanjing Forestry University, No. 159, LongPan Road, Nanjing 210037, People's Republic of China Full list of author information is available at the end of the article
}

and the overhead for collecting required information have significant effects on the system outage probability. Songtao Guo et al. [14] have considered applying a simultaneous wireless information and power transfer technique to cooperative clustered wireless sensor networks, they develop a distributed iteration algorithm of power allocation, power splitting, and relay selection to maximize the system energy efficiency, and they find that power splitting ratio plays an imperative role in the relay selection. Weikai Xu et al. [15] have proposed a power splitting-based relaying and a time switchingbased relaying protocols to enable wireless information transferring and energy harvesting in a denoise and forward two-way relay network, they analyze the two proposed protocols and obtain the optimal results for power of sources, power splitting ratio, and time switching factor. Zhe Wang et al. [16] have considered an energy harvesting relay station to improve the service quality of a cellular network. The goal is to assign each mobile station a relay station to minimize the probability of relay service outage. C. Huang et al. [17] proposed a deterministic EH model for the Gaussian relay channel and studied delay \& nondelay constrained traffics. $\mathrm{H}$. Li et al. [18] addressed the problem of transmission 
scheduling in EH-WSN when only partial information is available. I. Krikidis et al. [19] researched the interaction between data and energy queues when only knowledge of the arrival rates was available. Especially, K. Singh et al. [20] studied a joint source and relay transmitting power allocation scheme to maximize the system throughput. However, to the best of authors' knowledge, joint relay selection and power allocation for a cooperative communication clustering EH-WSN has not been addressed in the literature.

In this paper, we present a novel heuristic algorithm to further reduce the communication cost in a clustering EHWSN, where the saved energy can be use in data acquisition and processing. Cluster-based technique is especially popular in wireless sensor network where always dense nodes distributed: it divides the whole network into some clusters, while cooperative communication technique uses some relay nodes to enhance the transmission performance and reduce the whole energy consumption. Our proposed algorithm just chooses the best suitable relay node in the cluster and compute its transmitting power based on the channel condition and energy sustainability. The main contributions of our paper are summarized as follows:

(1) Based on the cooperative communication protocol in clustering EH-WSN, we propose a simplified cooperative relay model and analysis node's transmission power, bit error rate, and energy harvesting evolution procedure.

(2) To maximizing the energy utilization efficiency, we deduce a joint power allocation and relay selection algorithm. Then, we analyze the changes of energy saving efficiency and average network energy when different relays are chosen. These derivations provide practical design insights into the effect of various parameters on the system performance.

(3) The numerical results show that locating the relay node closer to the middle of source node and destination node yields more energy savings. It also demonstrates that the proposed cooperative protocol can improve the balance of cluster nodes' residual energy, since it intelligently track the node's energy sustainability in an online fashion.

The remainder of this paper is organized as follows. Section 2 presents the overview of system communication model; Section 3 analyzes the energy model; Section 4 introduces our joint optimization algorithm; Section 5 presents the experimental results including several key metrics; and Section 6 concludes the paper with drawback and future work.

\section{System communication model and analysis}

In this study, we mainly focus on the analysis and calculation of cooperative communication in EH-WSN, the cluster-routing protocol is not our main research target. So, we choose the energy harvesting genetic-based unequal clustering-optimal adaptive performance routing (EHGUC-OAPR) protocol [21] as our research basis instead. EHGUC-OAPR is an unequal clustering routing protocol: clusters which are closer to the base station (BS) have smaller size. Apparently, it can balance the energy consumption of the entire network and improve the data delivery ratio effectively. On the other hand, cooperative communications in such clustering EH-WSN are easier to understand: the dash-dotted lines represent the boundaries of clusters, and the black-colored nodes denote the cluster heads, while the straight lines with arrow are data transmission procedures, as is shown in Fig. 1.

The transmission procedure that a $\mathrm{EH}$ node transmits its data to the BS can be described as follows: first, a EH node shares the data to its cluster head; then, the cluster head selects an optimum cooperating relay within its cluster to collaboratively transmit the data to the next hop (i.e., another cluster head); finally, the cluster head transmissions are completed by a multi-hop scheme, and the sink (or BS) is the final destination. From the above diagram, we can simplify the network model as Fig. 2 for easy analysis. In which $R_{i}$ represents the optimum relay, $S$ is the source cluster head, and $D$ is the destination cluster head. All nodes in the network have $\mathrm{EH}$ ability and have only one antenna.

The specific cooperative communication procedure consists of two transmission slots: in the first slot, source cluster head $S$ broadcasts its data as byte $x$, all the relay node $R_{i}$ and destination cluster head $D$ can hear this message, and their received signals are shown as follow, respectively:

$$
\begin{aligned}
& y_{S, D}=\sqrt{P_{S}} h_{S, D} x+n_{S, D} \\
& y_{S, R_{i}}=\sqrt{P_{S}} h_{S, R_{i}} x+n_{S, R_{i}}
\end{aligned}
$$

$P_{S}$ just represents the transmission power of $S ; h_{S, D} \sim C$ $N\left(0, \sigma_{S, D}^{2}\right)$, and $h_{S, R_{i}} \sim C N\left(0, \sigma_{S, R_{i}}^{2}\right)$ are zero mean circularly symmetric complex Gaussian random variables, represent the independent identically distributed fading channel gains of $S$ to $D$ and $S$ to $R_{i}$. (p.s. all channels are orthogonal and irrelevant, its fading gain from node $i$ to node $j$ is $h_{i, j}$ which follows Rayleigh distribution), and $n_{S, D}$, and $n_{S, R_{i}}$ represent the additive Gaussian noise of $S$ to $D$ and $S$ to $R_{i}$, which also follows $C N\left(0, N_{0}\right)$.

Then follows the second slot: the selected optimum relay transfers the signal received in the first slot in a decode-and-forward [22] scheme, it will transmit at power $P_{R}$ if the decoding program is successful, or otherwise, it should keep silence. So in the end, $D$ should receive two signals: one direct from $S$ and the other from $R_{i}$. After a maximal ratio combining procedure, the recovered signal should be 


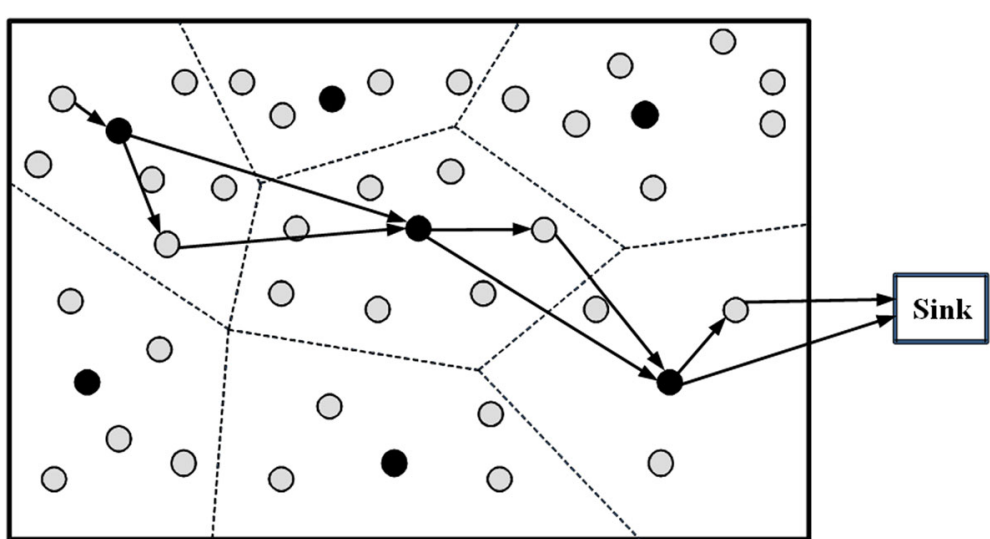

Fig. 1 Diagram of cooperative communication in the clustering protocol

$$
y_{S, D}^{\prime}=\sqrt{P_{S}} h_{S, D} y_{S, D}+\sqrt{P_{R}} h_{R_{i}, D} y_{R_{i}, D}
$$

It is worth noting that the overall end-to-end BER (bit error rate) of our cooperative system, $B R_{\text {coop }}$, may be expressed as the weighted sum of the BERs of these two cooperative cases:

$$
\begin{aligned}
\mathrm{BER}_{\text {coop }}= & \mathrm{BLER}_{S R} \cdot \mathrm{BER}_{\text {non-coop }} \\
& +\left(1-\mathrm{BLER}_{S R}\right) \cdot \mathrm{BER}_{\text {full-coop }}
\end{aligned}
$$

where $\mathrm{BLER}_{\mathrm{SR}}$ is the block error rate (BLER) of $S$ to $R_{i}$, BER non-coop is the BER of noncooperative transmission which directs from $S$ to $D$, and $\mathrm{BER}_{\text {full - coop }}$ is the BER of the cooperative diversity transmission which from $S$ and $R_{i}$ to $D$.

\section{Energy model and analysis}

Here, we consider the energy model of each node as

$$
E_{i}(\tau)=E_{i}(\tau-1)+P_{\mathrm{EH}, i}(\tau)-I\left(a_{i}(j)\right)\left(E_{T X}+E_{R X}\right)
$$

$E_{i}(\tau)$ is the residual energy of node $i$ at the end of time slot $\tau, P_{\mathrm{EH}, i}(\tau)$ is the harvested energy of node $i$ during slot $\tau, I(\cdot)$ is the binary indicator function and $a_{i}(j)$ is the event that node $i$ receives and transmits packets, and $E_{T X}, E_{R X}$ just represent the energy consumption of data transmission and reception.
The total average power consumption of a short-range communication system may be expressed as the sum of the total power consumption of the RF power amplifiers $P_{\text {PA }}$ and the total power consumption of all other transceiver circuit blocks $P_{\mathrm{CCT}}$. In which $P_{\mathrm{PA}}$ could be calculated by the node's transmission power $P_{\text {out }}$ :

$$
P_{\text {out }}=L E_{b} R_{b}
$$

where $E_{b}$ is the required energy per bit at the receiver for a target BER $P_{b} ; R_{b}$ is the bit rate; and $L$ represents the channel path loss and may be calculated according to $L=d_{i, j}^{k} L_{\mathrm{ref}}: d_{i, j}$ is the distance between node $i$ and $j, k$ is the path loss index, and $L_{\text {ref }}$ is the reference path loss when distance is $1 \mathrm{~m}$. So $P_{\mathrm{PA}}$ could be written as

$$
P_{\mathrm{PA}}=(\xi / \eta) P_{\mathrm{out}}
$$

$\eta$ is the drain efficiency of RF amplifier; $\xi$ is the peak to average power ratio decided by modulation scheme.

As for $P_{C C T}$, it consists of the transmission circuit power consumption $P_{\mathrm{CCT}-t x}$ and reception circuit power consumption $P_{\mathrm{CCT}-r x}$, exact values to be decided by detailed hardware components.

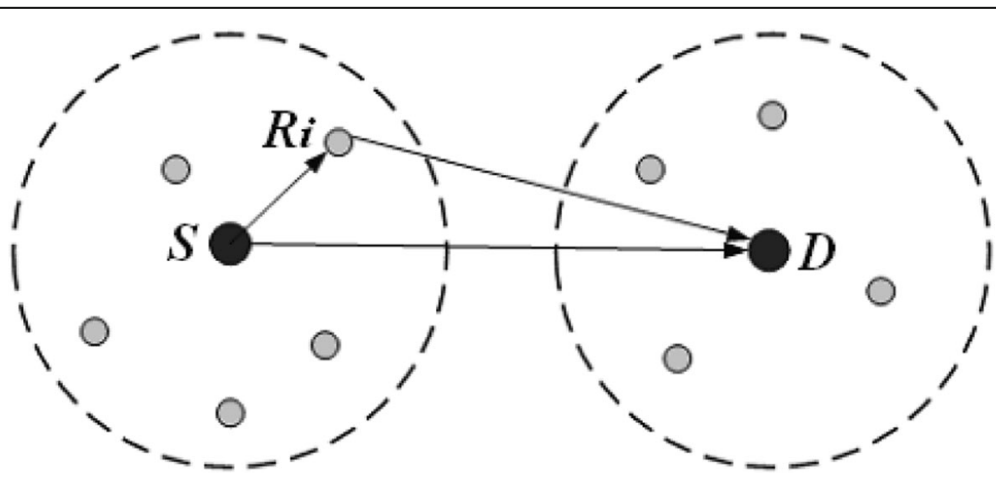

Fig. 2 Simplified cooperative relay network 
Thus, we can deduce the energy consumption per bit when working in conventional noncooperative communication status:

$$
E_{\text {non-coop }}=\left(P_{\mathrm{PA}-\text { source-non-coop }}+P_{\mathrm{CCT}-t x}+P_{\mathrm{CCT}-r x}\right) / R_{b}
$$

$P_{\mathrm{PA}-\text { source - non-coop }}$ is just the transmission power of source cluster head $S$.

Otherwise, if we call on the cooperative communication mode, the energy consumption per bit of $S$ is

$$
E_{\text {coop-source }}=\left(P_{\mathrm{PA} \text {-source-coop }}+P_{\mathrm{CCT}-t x}+2 P_{\mathrm{CCT}-r x}\right) / R_{b}
$$

and the consumption of relay node is

$$
\begin{aligned}
E_{\text {coop-relay }}= & \left(1-\mathrm{BLER}_{S R}\right) \\
& \times\left(P_{\mathrm{PA}-\text { relay-coop }}+P_{\mathrm{CCT}-t x}+P_{\mathrm{CCT}-r x}\right) / R_{b}
\end{aligned}
$$

$P_{\mathrm{PA} \text { - source-coop }}$ and $P_{\mathrm{PA}-\text { relay - coop }}$ are the transmission power of $S$ and $R_{i}$, respectively. So, the total transmission energy consumption per bit under cooperative diversity is

$$
E_{\text {coop }}=\left[\begin{array}{l}
P_{\mathrm{PA}-\text { source-coop }}+\left(1-\mathrm{BLER}_{S R}\right) P_{\mathrm{PA}-\text { relay-coop }} \\
+\left(2-\mathrm{BLER}_{S R}\right) P_{\mathrm{CCT}-t x}+\left(3-\mathrm{BLER}_{S R}\right) P_{\mathrm{CCT}-r x}
\end{array}\right] / R_{b}
$$

and the corresponding energy saving efficiency is

$$
\varepsilon_{\text {saving }}=100 \cdot\left(E_{\text {non-coop }}-E_{\text {coop }}\right) / E_{\text {non-coop }}
$$

When comparing formulas 8 and 11, it is apparently that the energy saving brings by cooperative communication must be greater than the energy cost increment in the meantime, only this can make $\varepsilon_{\text {saving }}$ be positive. In addition, the transmission energy consumption is proportional to the communication distance, so only the distance between $S$ and $D$ exceeds a certain threshold, the cooperative communication can be applicable. As for our EH-WSN, cluster heads are usually quite distant. Hence, the cooperative technique fits perfectly.

\section{Joint optimization algorithm design}

In this section, we present a joint power allocation and relay selection algorithm to optimize the energy utilization in the EH-WSN. It mainly possesses these functions: every source cluster head could choose the optimum relay node automatically and optimize their corresponding transmitting power. But for an EH-WSN, every EH node has its unique "energy neutral" operation requirement: the energy harvested in a time duration must be greater than the energy cost in the mean time, or else the node will turn down to sleep until enough energy has been acquired. So under the cooperative communication circumstance, the elected optimal relay node should meet two demands: both maximizing the saving efficiency bringing by cooperative diversity and equipped with the strongest energy sustainability. We can express it as the following function:

$$
\begin{gathered}
\underset{i, E_{S-T X}, E_{R-T X}}{\arg \max }\left\{\alpha \cdot S T(i)+(1-\alpha) \varepsilon_{\text {saving }}(i)\right\}, \\
\text { Subject to }\left\{\begin{array}{c}
\mathrm{BER}_{\text {coop }} \leq P_{b} \\
\forall i=1,2, \cdots, n: E_{i}\left(t_{\text {stop }}\right) \geq E_{i}\left(t_{\text {start }}\right)
\end{array}\right.
\end{gathered}
$$

where $\alpha$ is a proportionality coefficient, aiming at balancing the proportion of above two items in the objective function. $S T(i)$ is a representation of energy sustainability for EH node $i[21]$ :

$$
\begin{aligned}
S T(i) & =\frac{E_{M, i}}{\left(P_{\mathrm{EH}, i}+\sigma\right) \log \mu}\left(\mu^{\lambda(i)}-1\right), \quad \text { where } \\
\lambda(i) & =\frac{E_{M, i}-E_{S, i}}{E_{M, i}}
\end{aligned}
$$

$\sigma$ and $\mu$ are the appropriately chosen constants, $P_{\mathrm{EH}, i}$ is the harvesting power rate for each node $i, E_{S, i}$ and $E_{M, i}$ are the stored energy in the node $i$ 's battery and the maximum battery capacity, respectively. $S T(i)$ and $\varepsilon_{\text {saving }}(i)$ are the normalization results of $S T(i)$ and $\varepsilon_{\text {saving. }} i$ is the optional relay node in the cluster. $E_{S-T X}$ and $E_{R-T X}$ are the transmit energies per information bit of the nodes $S$ and $R$, respectively.

$$
\begin{aligned}
& E_{S-T X}=(\eta / \xi) P_{\mathrm{PA}-\text { source-coop }} / R_{b} \\
& E_{R-T X}=(\eta / \xi) P_{\mathrm{PA}-\text { relay-coop }} / R_{b}
\end{aligned}
$$

Through the analysis of formula 14, we can figure that $S T(i)$ is generating by nodes in the cluster automatically, which varies with the environment's energy. As for the $\varepsilon_{\text {sav- }}$ ing, from formula 12, we could know, when node $S$ and node $D$ are chosen; $\varepsilon_{\text {saving }}$ is in inverse proportion to $E_{\text {coop. }}$. Hence, in order to maximize $\varepsilon_{\text {saving, }}$, we just need to minimize $E_{\text {coop }}$. So, the next paragraphs describe the optimization of $E_{\text {coop: }}$ :

For the sake of easy analysis, we take $E_{S-T X}$ and $E_{R-T X}$ to represent the constraints in formula 13. Firstly, we use $\left(\mathrm{SNR}_{S R}\right.$ and $\left.\mathrm{SNR}_{R D}\right)$ to calculate $\left(B E R_{\text {non - coop }}\right.$ and $\left.B E R_{\text {full - coop }}\right)[23]$ :

$$
\begin{aligned}
& \mathrm{BER}_{\text {non-coop }} \approx 1 /\left(4 \mathrm{SNR}_{R D}\right), \\
& \mathrm{BER}_{\text {full-coop }} \approx 3 /\left(16 \mathrm{SNR}_{S D} \cdot \mathrm{SNR}_{R D}\right)
\end{aligned}
$$

where $\mathrm{SNR}_{S R}$ and $\mathrm{SNR}_{R D}$ are the signal noise ratio of communication channel from node $S$ to relay $R$ and from $R$ to $D$. 
Table 1 Simulation parameters

\begin{tabular}{llll}
\hline Parameter & Value & Parameter & Value \\
\hline Target end-to-end BER $P_{b}$ & $10^{-3}$ & Bit rate $R_{b}$ & $10 \mathrm{~kb} / \mathrm{s}$ \\
Target source-relay BER $P_{s r}$ & $10^{-4}$ & Transmission power $P_{\text {CCT }-t x}$ & $98.2 \mathrm{~mW}$ \\
Path loss exponent $k$ & 3.5 & Noise power spectral density $N_{0}$ & $-171 \mathrm{dBm} / \mathrm{Hz}$ \\
Peak average ratio $\xi$ & 1 & Reception power $P_{\mathrm{CCT}-r x}$ & $109.5 \mathrm{~mW}$ \\
Drain efficiency of RF $\eta$ & 0.35 & Energy harvesting rate $P_{\mathrm{EH}, n}$ & $1 \mathrm{~mW}$ \\
$\sigma, \mu$ & $0.1,5$ & Reference path loss $L_{\text {ref }}$ & $10^{9}(90 \mathrm{~dB})$ \\
\hline
\end{tabular}

Secondly, we also calculate BLER $\mathrm{R}_{S R}$ as follow:

$$
\mathrm{BLER}_{S R} \approx K / \mathrm{SNR}_{S R}
$$

Of which $K$ is a scaling factor. And according to formula 6,15 , and 16 , we can infer that

$$
\begin{aligned}
& \mathrm{SNR}_{S R}=\left(E_{S-T X} / N_{0}\right) / L_{S R} \\
& \mathrm{SNR}_{S D}=\left(E_{S-T X} / N_{0}\right) / L_{S D} \\
& \mathrm{SNR}_{R D}=\left(E_{R-T X} / N_{0}\right) / L_{R D}
\end{aligned}
$$

where $L_{S R}, L_{S D}$, and $L_{R D}$ show the communication channel path loss for node $S$ to relay $R$, node $S$ to $D$, and node $R$ to $D$. For convenience of analysis, we take these three as given parameters which could be calculated by each node.
Then, we substitute formulas $17-22$ into formula 4 , it can be derived as

$$
\mathrm{BER}_{\text {coop }}=\frac{\left(1-\frac{K N_{0} L_{S R}}{E_{S-T X}}\right) \cdot 3 \cdot N_{0}^{2} \cdot L_{S D} \cdot L_{R D}}{16 \cdot E_{S-T X} \cdot E_{R-T X}}+\frac{K \cdot N_{0}^{2} \cdot L_{S D} \cdot L_{S R}}{4 \cdot E_{S-T X}^{2}}
$$

As well as $E_{\text {coop }}$ can be expressed as

$$
\begin{aligned}
E_{\text {coop }}= & (\xi / \eta) E_{S-T X}+\left(1-\frac{K N_{0} L_{S R}}{E_{S-T X}}\right) \cdot(\xi / \eta) E_{R-T X} \\
& +\left(2-\frac{K N_{0} L_{S R}}{E_{S-T X}}\right) \cdot \frac{P_{\mathrm{CCT}-t x}}{R_{b}}+\left(3-\frac{K N_{0} L_{S R}}{E_{S-T X}}\right) \cdot \frac{P_{\mathrm{CCT}-r x}}{R_{b}}
\end{aligned}
$$

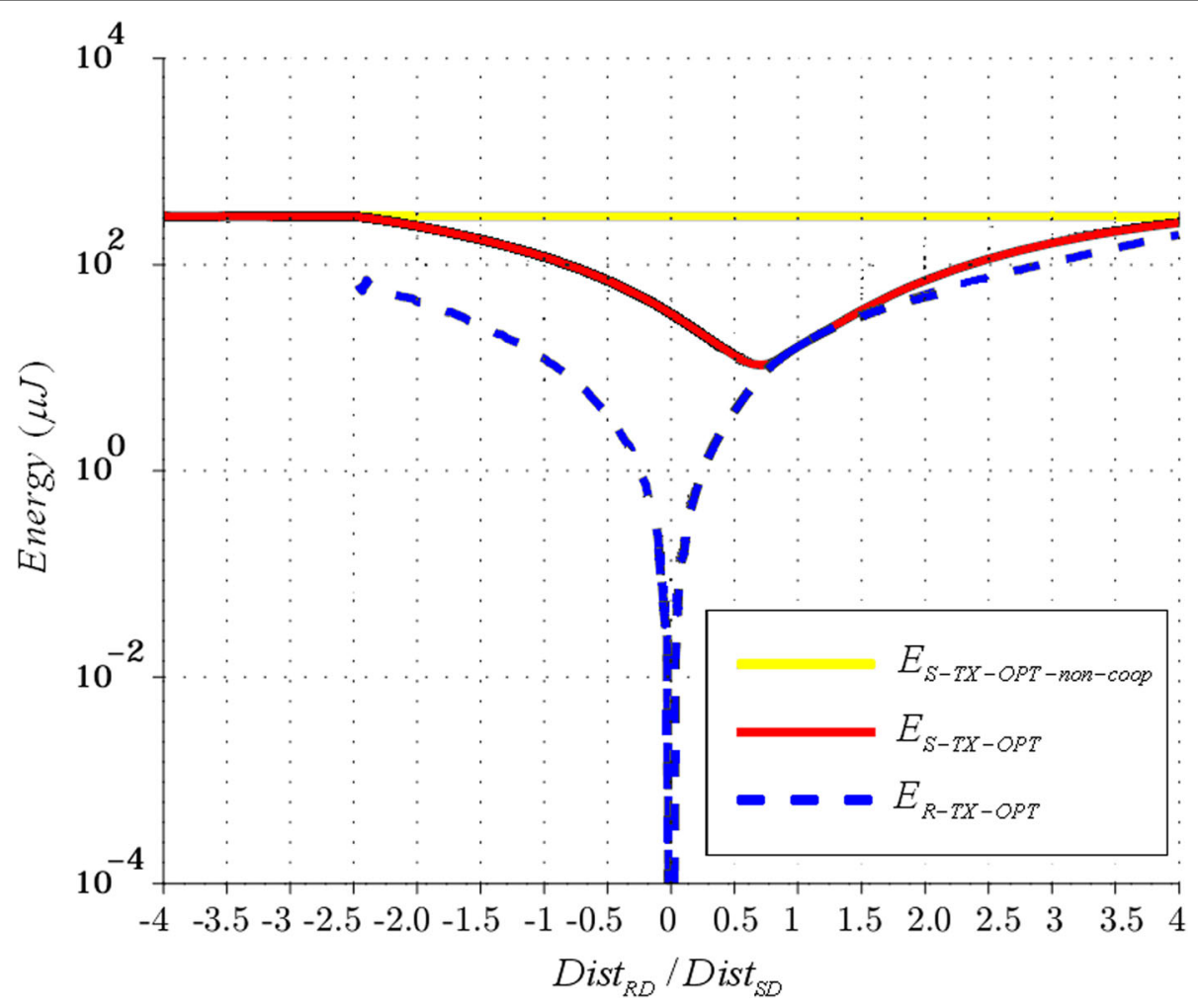

Fig. 3 Energy variance curves when relay node is moving 


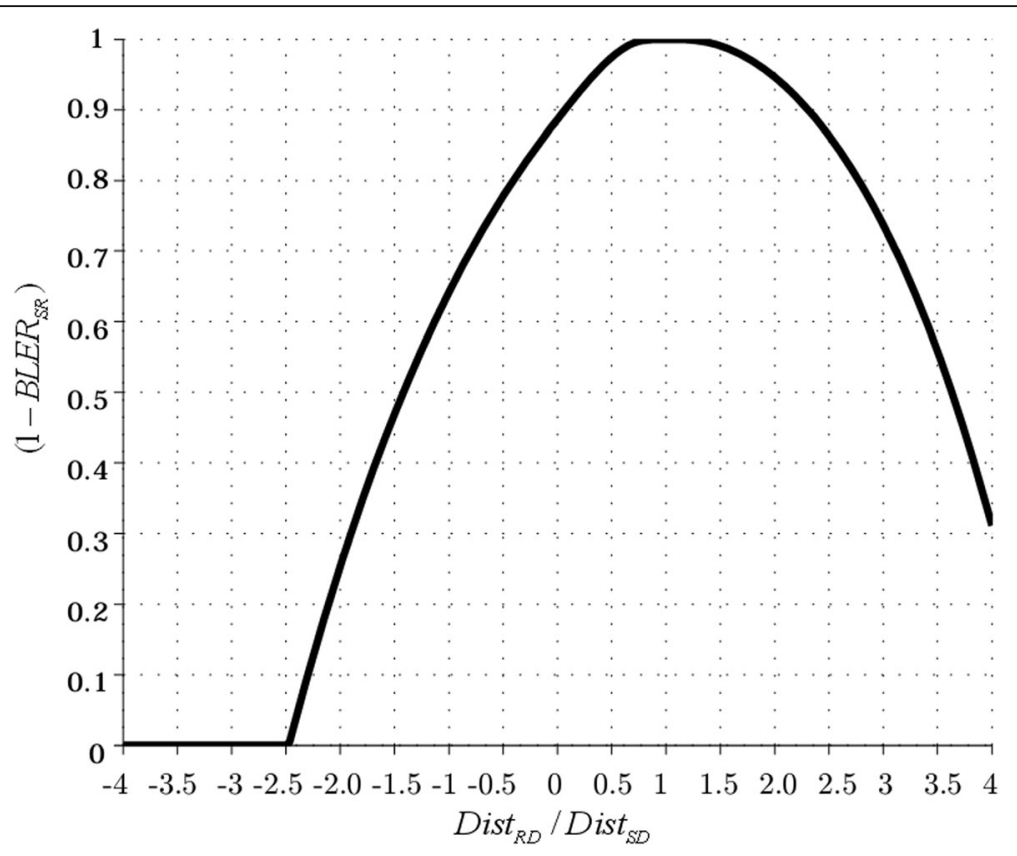

Fig. 4 The data relay success rate vs. location changes of $R$

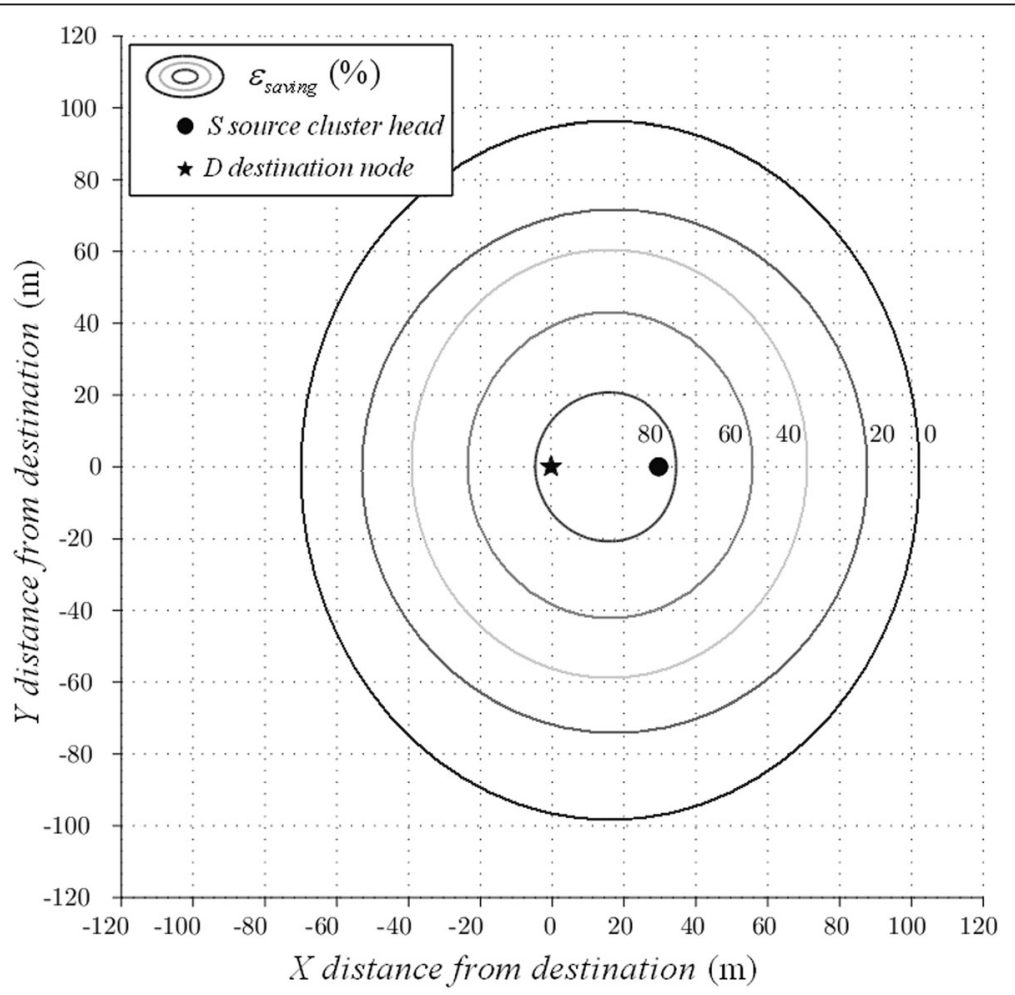

Fig. 5 The energy saving efficiency $\varepsilon_{\text {saving }}$ vs. the distance from $R$ to $D$ 


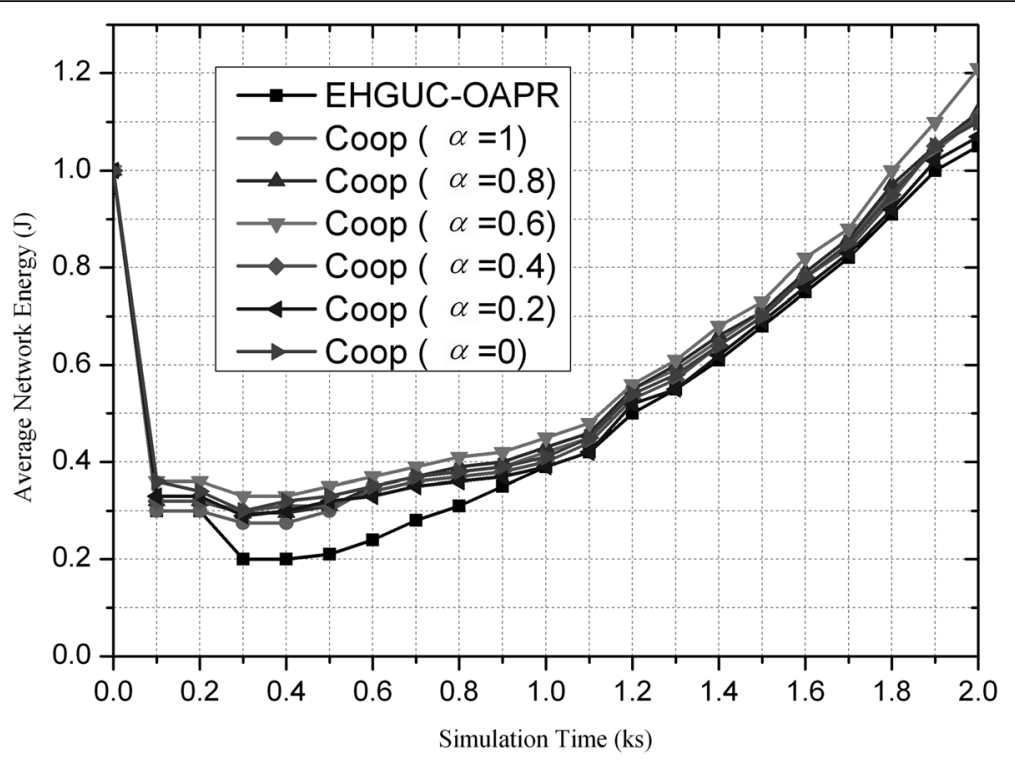

Fig. 6 Average network energy

Moreover, formula 23 can be presented below after taking in the constraints:

$$
E_{R-T X}=\frac{\left(1-\frac{K N_{0} L_{S R}}{E_{S-T X}}\right) \cdot 3 \cdot N_{0}^{2} \cdot L_{S D} \cdot L_{R D}}{16 \cdot E_{S-T X} \cdot\left(P_{b}-\frac{K N_{0}^{2} L_{S D} L_{S R}}{4 \cdot E_{S-T X}^{2}}\right)}
$$

In this way, finally, we can translate the function of $E_{\text {coop }}$ minimization into a single variable optimization algorithm with $E_{S-T X}$ :

$$
\arg \min E_{S-T X}\left\{\begin{array}{c}
E_{S-T X}+\frac{\left(1-\frac{K N_{0} L_{S R}}{E_{S-T X}}\right) \cdot 3 \cdot N_{0}^{2} \cdot L_{S D} \cdot L_{R D}}{16 \cdot E_{S-T X} \cdot\left(P_{b}-\frac{K N_{0}^{2} L_{S D} L_{S R}}{4 \cdot E_{S-T X}^{2}}\right)} \\
+\frac{\eta}{\xi}\left[\left(2-\frac{K N_{0} L_{S R}}{E_{S-T X}}\right) \cdot \frac{P_{\mathrm{CCT}-t x}}{R_{b}}+\left(3-\frac{K N_{0} L_{S R}}{E_{S-T X}}\right) \cdot \frac{P_{\mathrm{CCT}-\mathrm{rx}}}{R_{b}}\right]
\end{array}\right\}
$$

Obviously, the above formula is a nonlinear function of $E_{S-T X}$; we can use an exhaustion method to compute the optimal result, but at the same time, it must cause a heavy computation burden to the sensor node, in order

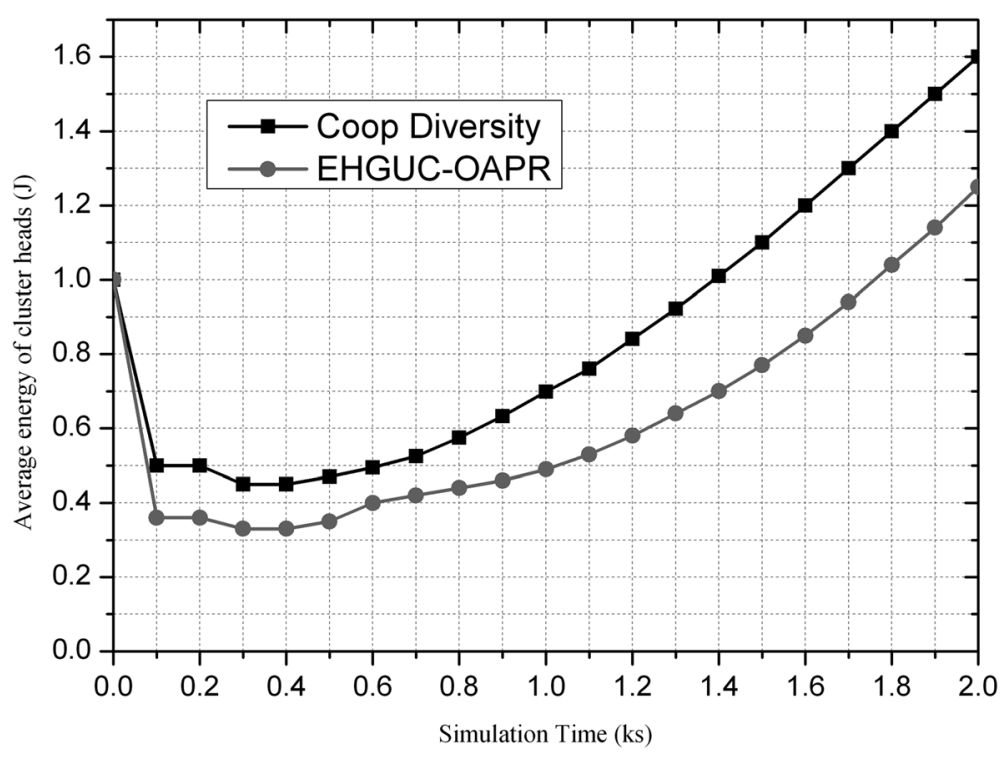

Fig. 7 Contrast of average energy of cluster heads $(a=0.6)$ 


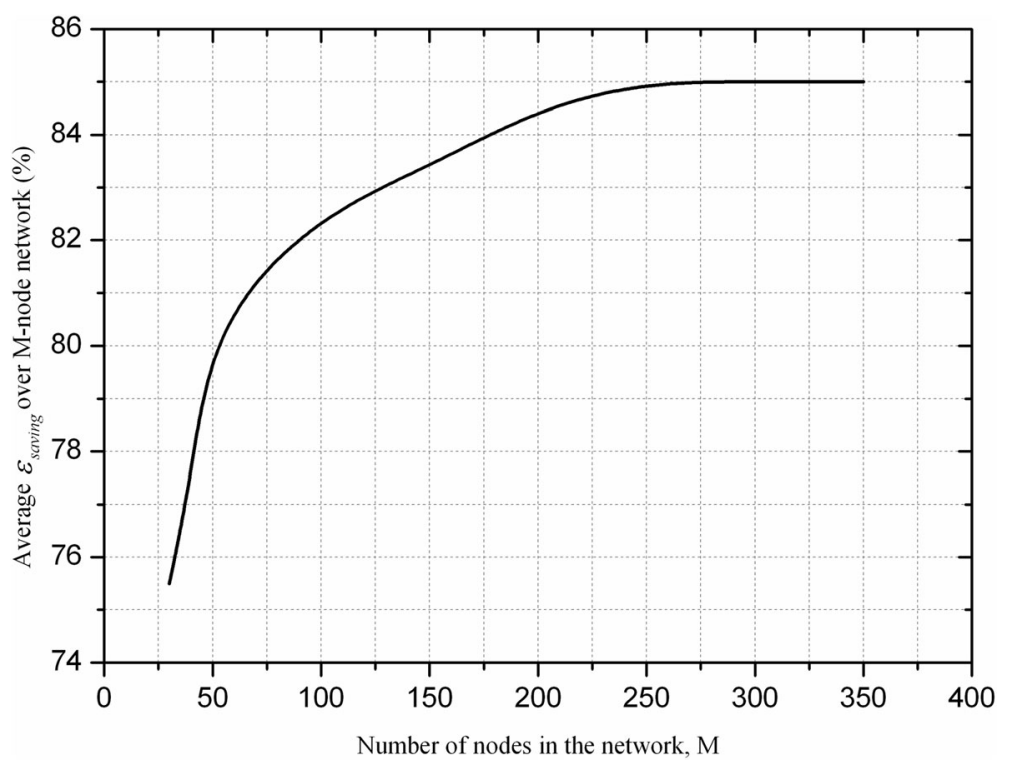

Fig. 8 Average energy saving vs. EH-WSN with different number nodes

to avoid this, we make a further analysis on formula 26 : when node $S$ and $D$ have been chosen already, $L_{S D}$ should be seen as a constant value; thus, we only have to deal with $L_{S R}$ and $L_{R D}$. So, the optimization problem can be approximately decomposed as a combination of these two parameters' independent actions.

If we regard $L_{S R}$ as zero, formula 26 is shown as

$$
\lim _{L_{S R \rightarrow 0}} \underset{E_{S-T X}}{\arg \min }\left\{E_{\text {coop }}\right\}=\sqrt{\frac{3 \cdot N_{0}^{2} \cdot L_{S D} \cdot L_{R D}}{16 \cdot P_{b}}}
$$

Else if we regard $L_{R D}$ as zero, it is shown as

$$
\lim _{L_{R D \rightarrow 0}} \underset{E_{S-T X}}{\arg \min }\left\{E_{\text {coop }}\right\}=\sqrt{\frac{k \cdot N_{0}^{2} \cdot L_{S R} \cdot L_{S D}}{4 \cdot P_{b}}}
$$

Therefore, the optimization problem can be optimally represented as the joint action of formulas 27 and 28:

$$
\begin{aligned}
E_{S-T X-\mathrm{OPT}} & =\lim _{L_{S R \rightarrow 0}} \underset{E_{S-T X}}{\arg \min }\left\{E_{\text {coop }}\right\}+\lim _{L_{R D \rightarrow 0}} \arg \min \left\{E_{E_{S-T X}}\right\} \\
& =\sqrt{\frac{3 \cdot N_{0}^{2} \cdot L_{S D} \cdot L_{R D}}{16 \cdot P_{b}}}+\sqrt{\frac{k \cdot N_{0}^{2} \cdot L_{S R} \cdot L_{S D}}{4 \cdot P_{b}}}
\end{aligned}
$$

And more:

$$
E_{R-T X-\mathrm{OPT}}=\frac{\left(1-\frac{K N_{0} L_{S R}}{E_{S-T X-\mathrm{OPT}}}\right) \cdot 3 \cdot N_{0}^{2} \cdot L_{S D} \cdot L_{R D}}{16 \cdot E_{S-T X-\mathrm{OPT}} \cdot\left(P_{b}-\frac{K N_{0}^{2} L_{S D} L_{S R}}{4 \cdot E_{S-T X-\mathrm{OPT}}^{2}}\right)}
$$

So far, we have worked out the transmitting power allocation equations for every distributed feasible relay node. Each candidate relay node in the clusters could calculate its corresponding transmitting power based on the $L_{S R}$ and $L_{R D}$ according to formulas 29 and 30; also the energy saving efficiency $\varepsilon_{\text {saving }}$ could be figured out.

At last, we know that every candidate relay node could calculate both $\varepsilon_{\text {saving }}(i)$ and $S T(i)$ automatically and independently; hence, a relay index $T_{R E}$ can be defined as

$$
T_{R E}(i)=\alpha \cdot S T(i)+(1-\alpha) \varepsilon_{\text {saving }}(i)
$$

Furthermore, the cluster head is responsible for collecting each candidate's $\varepsilon_{\text {saving }}(i)$ and $S T(i)$ and computing the $T_{R E}(i)$. The one with maximal value just become the cooperative relay. But also it should be noted that if

\begin{tabular}{|c|c|c|c|c|c|c|c|c|c|c|c|c|}
\hline Dist $_{R D} /$ Dist $_{S D}$ & -2.0 & -1.5 & -1.0 & -0.5 & 0 & 0.5 & 1.0 & 1.5 & 2.0 & 2.5 & 3.0 & 3.5 \\
\hline Difference $(\mu \mathrm{J})$ & 10.1 & 7.8 & 6.4 & 4.3 & 2.5 & 1.3 & 2.6 & 4.4 & 6.4 & 7.9 & 10 & 12.3 \\
\hline
\end{tabular}
a node's current energy status cannot afford the cooperative communication, it would not be selected into the candidate set. The diagram of this joint optimization algorithm is shown below.

Table 2 Comparison between the suboptimal results and the optimal results 
Algorithm 1 Joint power allocation and relay selection optimization algorithm

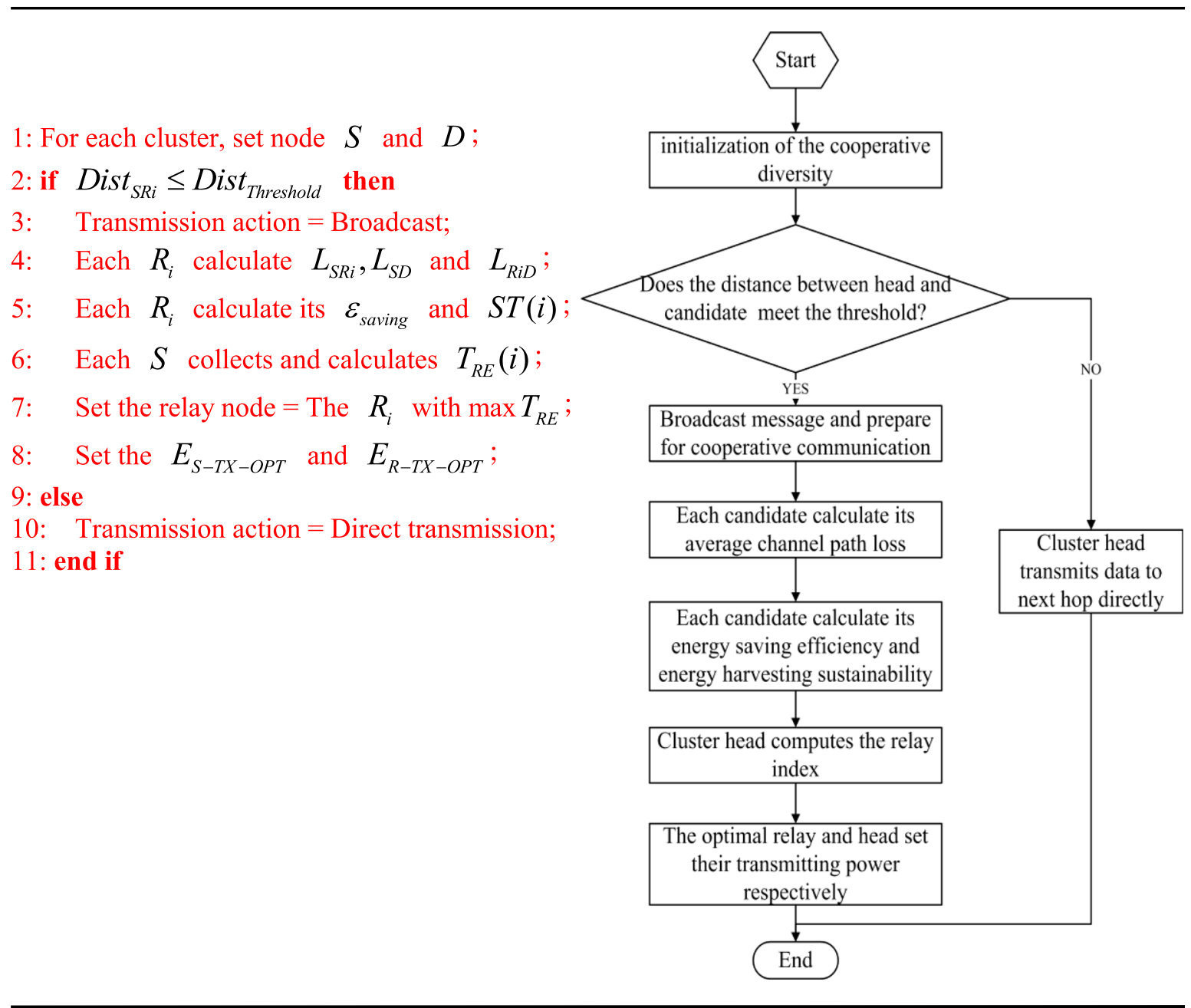

\section{Results and analysis of simulation}

In this section, we investigate the performance of our optimization algorithm via MATLAB. An EHWSN with 200 nodes can be assumed to be deployed in a monitoring area of $500 \times 500 \mathrm{~m}$, the routing protocol "EHGUC-OAPR" has already loaded and the clusters have formed up either, then our experiment carries out. Here, some detailed parameters are shown in Table 1.

First, we do some research on the performance of transmitting power allocation block. We assume a onedimensional situation that the source cluster head $S$, relay $R$, and destination node $D$ lie in a straight line, in which $S$ location is regarded as original point, and the distance from $D$ to $S$ is $30 \mathrm{~m}$; by readjusting the location of $R$ gradually, we can obtain the numerical changes of optimal power allocation. Simulation result is shown in Fig. 3.

The yellow curve displays the source head's transmitting energy when in noncooperative situation; it apparently keeps the same because the relay does not work. The red and blue curves show the source head's and relay's transmitting energy under cooperation, respectively; Dist $_{R D}$ is just the distance from $R$ to $D$, when it becomes zero means $R$ coincides with $D$. From the above result, we can see that the total transmission energy $\left(E_{S-T X-}\right.$ OPT plus $\left.E_{R-T X-O P T}\right)$ falls to minimum when $R$ is placed in the middle of $S$ and $D\left(\right.$ Dist $_{R D} /$ Dist $\left._{S D} \approx 0.5\right)$; otherwise, it rises up rapidly. In addition, Fig. 4 shows the effective operation distance threshold of relay node $\left(-2.5<\right.$ Dist $_{R D} /$ Dist $\left._{S D}<3.5\right)$, and the transmitting energy of $S$ takes a greater proportion. P.S. the minus sign 
means $R$ is located on the other side of $S$ opposite direction with $D$.

Furthermore, the numerical value variation of BLER $_{S R}$ at the relay node is also recorded in Fig. 4: it represents the data reception success rate from $S$ to $R$. We can see that $R$ cannot decode data correctly when Dist $_{R D} /$ Dist $_{S D}<-2.5$.

Then, we turn to a two-dimensional situation, where relay can move on a plane surrounded by $S$ and $D$. Here, $D$ is placed on the original point with $S 30 \mathrm{~m}$ away. The corresponding energy saving efficiency $\varepsilon_{\text {saving }}$ is calculated and shown in Fig. 5. Five contour lines represent different energy saving levels when $R$ is located inside. In macroscale, $R$ 's position seems nearly a circle under the same $\varepsilon_{\text {saving; }}$ but in microscale, the closer to the middle of $S$ and $D$, the more efficient working of $R$.

After these experiments, we carry our joint optimization algorithm into EHGUC-OAPR protocol, where the initial energy for all nodes is $1 \mathrm{~J}$. We calculate the network average residual energy status as shown in Fig. 6. We can find that every cooperative scheme can reach a better energy status than noncooperative scheme, and the whole EH-WSN reach the energetic optimum when $\alpha=0.6$.

Moreover, we examine the energy variation of every cluster head, as shown in Fig. 7. It is obvious that cooperative communication could greatly improve the energy efficiency of EH-WSN, hence saving more power to do other tasks, such as data acquisition and processing.

Additionally, we modify the nodes' total number in the EH-WSN, and we evaluate the average $\varepsilon_{\text {saving }}$ of each scenario, as shown in Fig. 8. We can see that the network with bigger total number has higher efficiency $\varepsilon_{\text {saving. }}$. The reason is the more nodes in a cluster, the higher probability of a bigger $T_{R E}(i)$.

Finally, we try to solve the equation 26 and compare the results with our proposed suboptimal results, and we proceed within the one-dimensional circumstance. The difference of total transmission energy $\left(E_{S}-T X\right.$-OPT plus $\left.E_{R-T X-\mathrm{OPT}}\right)$ between them is shown in Table 2 .

We could see that the difference falls to the minimum also when relay $R$ is placed in the middle of $S$ and $D$, and the corresponding relative error is almost less than $5 \%$. So, the proposed optimization computing method is really available and has enough precision.

\section{Conclusions}

In this paper, we have investigated an optimal relay selection for energy efficient cooperation in a clustering based EH-WSN. We have formulated a novel and computationally efficient relay selection heuristics for sensor nodes based on both local path loss values and energy harvesting rates. Extensive simulation results are presented to demonstrate that the proposed algorithm achieves near optimally relay node selection and transmitting power allocation. Therefore, this scheme forms the basis of a simple and practical cooperation strategy for EH-WSN and can be directly deployed in real applications (such as solar power monitoring system). It also can be integrated with other optimization protocols to achieve energy efficient network-wide cooperation.

Future work will focus on the proposal of a multiobjective algorithm for node placement and coverage of cooperative EH-WSN. Moreover, we will consider different application cases, including energy harvester-sharing networks and structural health monitoring scene.

\section{Acknowledgements}

This work was supported by the Natural Science Foundation of Jiangsu Province, China (BK20150880). The authors also gratefully acknowledge the helpful comments and suggestions of the reviewers, which have improved the presentation.

\section{Competing interests}

The authors declare that they have no competing interests.

\section{Author details}

${ }^{1}$ College of Information Science and Technology, Nanjing Forestry University, No. 159, LongPan Road, Nanjing 210037, People's Republic of China. ${ }^{2}$ College of Automation, Nanjing University of Aeronautics and Astronautics, NO. 29 Yudao St., Nanjing 210016, People's Republic of China.

Received: 8 September 2016 Accepted: 25 January 2017

Published online: 06 February 2017

\section{References}

1. M Shin, I Joe, Energy management algorithm for solar-powered energy harvesting wireless sensor node for Internet of Things. IET Commun. 10(12), 1508-1521 (2016)

2. C Guo, B Liao, L Huang, Q Li, X Lin, Convexity of fairness-aware resource allocation in wireless powered communication networks. IEEE Commun. Lett. 20(3), 474-477 (2016)

3. D Del Testa, N Michelusi, M Zorzi, Optimal transmission policies for two-user energy harvesting device networks with limited state-of-charge knowledge. IEEE Trans. Wirel. Commun. 15(2), 1393-1405 (2016)

4. F lannello, O Simeone, U Spagnolini, Medium access control protocols for wireless sensor networks with energy harvesting. IEEE Trans. Commun. 60(5), 1381-1389 (2012)

5. L-O Varga, G Romaniello, M Vučinić et al., GreenNet: an energyharvesting IP-enabled wireless sensor network. IEEE Internet Things J. 2(5), 412-426 (2015)

6. THe, K-W Chin, S Soh, On wireless power transfer and max flow in rechargeable wireless sensor networks. IEEE Access 4, 4155-4167 (2016)

7. G Martinez, S Li, C Zhou, Wastage-aware routing in energy-harvesting wireless sensor networks. IEEE Sensors J. 14(9), 2967-2974 (2014)

8. S Lee, B Kwon, S Lee, AC Bovik, BUCKET: scheduling of solar-powered sensor networks via cross-layer optimization. IEEE Sensors J. 15(3), 1489-1503 (2015)

9. $\quad Y$ Tan, $X$ Yin, A dynamic scheduling algorithm for energy harvesting embedded systems. EURASIP J. Wirel. Commun. Netw. 114, 1-8 (2016)

10. D Noh, T Abdelzaher, Efficient flow-control algorithm cooperating with energy allocation scheme for solar-powered WSNs. Wireless Commun. Mobile Comput. 12(5), 379-392 (2012)

11. Y Dong, MJ Hossain, J Cheng, Performance of wireless powered amplify and forward relaying over Nakagami-\$m\$ fading channels with nonlinear energy harvester. IEEE Commun. Lett. 20(4), 672-675 (2016)

12. S Gong, L Duan, N Gautam, Optimal scheduling and beamforming in relay networks with energy harvesting constraints. IEEE Trans. Wirel. Commun. 15(2), 1226-1238 (2016)

13. K-H Liu, Performance analysis of relay selection for cooperative relays based on wireless power transfer with finite energy storage. IEEE Trans. Veh. Technol. 65(7), 1977-1987 (2016) 
14. S Guo, F Wang, Y Yang, B Xiao, Energy-efficient cooperative transmission for simultaneous wireless information and power transfer in clustered wireless sensor networks. IEEE Trans. Commun. 63(11), 4405-4417 (2015)

15. W Xu, Z Yang, Z Ding, L Wang, P Fan, Wireless information and power transfer in two-way relaying network with non-coherent differential modulation. EURASIP J. Wirel. Commun. Netw. 131, 1-10 (2015)

16. Z Wang, X Wang, M Aldiab, T Jaber, User association for energy harvesting relay stations in cellular networks. EURASIP J. Wirel. Commun. Netw. 264, 1-12 (2015)

17. C Huang, R Zhang, S Cui, Throughput maximization for the Gaussian relay channel with energy harvesting constraints. IEEE J. Sel. Areas Commun. 31(8), 1469-1479 (2013)

18. H Li, N Jaggi, B Sikdar, Relay scheduling for cooperative communications in sensor networks with energy harvesting. IEEE Trans. Wirel. Commun. 10(9), 2918-2928 (2011)

19. I Krikidis, T Charalambous, JS Thompson, Stability analysis and power optimization for energy harvesting cooperative networks. IEEE Signal Process Lett. 19(1), 20-23 (2012)

20. K Singh, K Meng-Lin, J-C Lin, Joint power allocation, equalization, and relay selection for MIMO relay networks with multipath receptions. IEEE Trans. Veh. Technol. 65(7), 5160-5174 (2016)

21. $Y$ Wu, L Wenbo, Routing protocol based on genetic algorithm for energy harvesting-wireless sensor networks. IET Wireless Sens. Syst. 3(2), 112-118 (2013)

22. AS Ibrahim, AK Sadek, S Weifeng, KJ Ray Liu, Cooperative communications with relay-selection: when to cooperate and whom to cooperate with? IEEE Trans. Wirel. Commun. 7(7), 2814-2827 (2008)

23. JG Proakis, M Salehi, Digital Communications, 5th edn. (McGraw-Hill, New York, 2007)

\section{Submit your manuscript to a SpringerOpen ${ }^{\circ}$ journal and benefit from:}

- Convenient online submission

- Rigorous peer review

- Immediate publication on acceptance

- Open access: articles freely available online

- High visibility within the field

- Retaining the copyright to your article

Submit your next manuscript at $\gg$ springeropen.com 